\title{
Labyrinthe
}

17 | 2004 (1)

Jacques Rancière, l'indiscipliné

\section{René Major (dir.), États généraux de la psychanalyse}

\section{Philippe Lacour}

\section{OpenEdition}

Journals

Édition électronique

URL : http://journals.openedition.org/labyrinthe/185

DOI : 10.4000/labyrinthe.185

ISSN : 1950-6031

Éditeur

Hermann

Édition imprimée

Date de publication : 15 avril 2004

Pagination : 119-121

Référence électronique

Philippe Lacour, «René Major (dir.), États généraux de la psychanalyse », Labyrinthe [En ligne], 17 | 2004 (1), mis en ligne le 13 juin 2008, consulté le 22 septembre 2020. URL : http://journals.openedition.org/ labyrinthe/185; DOI : https://doi.org/10.4000/labyrinthe.185

Propriété intellectuelle 


\title{
René MAJOR (dir.), États généraux de la psychanalyse, juillet 2000, Aubier, 2003
}

\author{
Philippe LACOUR \\ placour@netcourrier.com
}

Née voici un peu plus d'un siècle, la psychanalyse a été la cible de multiples attaques, mais a fini, malgré les incompréhensions positivistes rémanentes ${ }^{1}$, par s'imposer durablement dans le paysage intellectuel, non sans quelques dérives herméneutiques incontrôlées, où le manque de rigueur le dispute parfois au charlatanisme du concept et de la clinique. Ce n'est pas, en tout cas, le moindre mérite de ce petit livre, États généraux de la psychanalyse, juillet 2000, que de nous proposer de nous étonner à nouveau de tout ce qui fait l'étrangeté de cette discipline.

Ce recueil d'articles est le fruit d'un inévitable choix opéré parmi les quelques deux cent cinquante contributions de psychanalystes communiquées à Paris lors de ces premiers états généraux. Rappelons l'esprit de ce rassemblement. D'abord, son an-institutionnalisme (ou trans-institutionnalisme) - au contraire de tant de congrès marqués par le respect d'une certaine obédience (ou ligne) -, mais aussi son internationalisme, la constitution de groupes de travail, la création d'un site internet ${ }^{2}$, ainsi qu'un certain nombre de déclarations et de prises de position ${ }^{3}$; il s'agissait bien d'inaugurer une vaste dyna-

1. Voir par exemple les critiques formulées par Adolf Grünbaum, de l'école d'épistémologie de Pittsburg, (The Foundations of Psychoanalysis, a Philosophical Critique, University of California Press, BerkeleyLos Angeles-London, 1984; traduction française : Les Fondements de la psychanalyse, Paris, Puf, 1996), et Jacques Bouveresse (Philosophie, mythologie et pseudo-science, Wittgenstein lecteur de Freud, Nîmes, éditions de l'Éclat, 1991). Stephan Pohl, élève de Lorenzer (dont s'inspire Habermas dans Connaissance et Intérêt), s'est expliqué avec Grünbaum dans un livre récent et pas encore traduit en français: Wissenschaftstheoretische und methodologische Probleme der Psychanalyse. Eine Auseinandersetzung mit Grünbaums Psychoanalysekritik, Königshausen \& Neumann, 1991. Sur le rapport de Freud à Wittgenstein, voir le livre de Paul-Laurent Assoun: Freud et Wittgenstein, Paris, Puf, 1988.

2. http://www.psychanalyse.refer.org. La mémoire des Journées de Paris est consultable à l'adresse suivante: http://www.psychanalyse.refer.org/archivesparis2000.php

3. Voir, en annexe du recueil, la « déclaration sur la spécificité de la psychanalyse » (autonomie par rapport aux diverses formes de psychothérapies et aux pouvoirs publics), la " prise de position sur la cruauté et la peine de mort », la demande de création «d'écoles supérieures de psychanalyse », ou encore la constitution d'un « institut des hautes études en psychanalyse » (où se croiseraient les enseignements de la psychanalyse, de la médecine et de la psychiatrie, des sciences du vivant, de la philosophie et de la linguistique, de l'esthétique, du droit, de la sociologie, de l'anthropologie, de l'histoire enfin). 
mique de réflexion, collective et pérenne ${ }^{4}$. Ensuite, la revendication du sens politique de la métaphore: ces états généraux répondent à une situation de crise. Sur ce point, le consensus est très large, et le terme « crise » est un de ceux qui reviennent le plus souvent sous la plume des auteurs. Gilou Garcia Reinoso résume assez bien ce sentiment lorsqu'il dit que « les états généraux surgissent du malaise dans la psychanalyse »; René Major parle quant à lui de «situation de crise »; Jacques Derrida, des « états d'âme de la psychanalyse »; Joël Birman, de «crise profonde de la souveraineté psychanalytique ». Élisabeth Roudinesco ${ }^{5}$ 'est pas en reste, qui se risque même à un diagnostic: « Ce qu'on appelle désormais la crise de la psychanalyse n'est autre, à mon sens, qu'une crise liée à la définition de sa spécificité dans un monde, celui du $\mathrm{XXI}^{\mathrm{e}}$ siècle, où, d'ores et déjà, on assiste à un grand essor des psychothérapies : environ un millier recensées aujourd'hui. » Le paradoxe tient précisément au fait que cette crise est contemporaine d'une extension considérable des domaines d'intervention de la psychanalyse. Filons la métaphore politique: ces états généraux assument un désir de constitution (création d'un institut) et d'abolition des privilèges (la prévalence de certaines institutions); mais la différence avec 1789 tient à l'absence de roi (d'où une organisation décentrée).

Outre cette dimension institutionnelle permanente ${ }^{6}$, Michel Plon ${ }^{7}$, Gilou Garcia Reinoso ${ }^{8}$ et Armando Uribe ${ }^{9}$ soulignent la préoccupation politique, au sens large, de la psychanalyse ${ }^{10}$. Par ailleurs, plusieurs thèmes font l'objet d'une attention particulière et persistante. D'abord, le souci de résister intelligemment au réductionnisme, en étudiant précisément ses modalités, sans en rester à la critique naïve d'une caricature de la position adverse. Sur ce point, la communication d'Amy Cohen ${ }^{11}$ est très précise et utile, notamment pour sa différenciation du cognitivisme, du connexionisme et de l'enaction, correspondant aux trois rencontres possibles entre psychanalyse et neurosciences (métaphorique, littérale, dialogique). L'auteur a le mérite de tordre le cou à des préjugés antiscientistes encore répandus (par exemple, celui du prétendu

4. La seconde édition des États généraux a eu lieu à Rio de Janeiro, en octobre-novembre 2003.

5. «État de la psychanalyse dans le monde », dans États généraux de la psychanalyse, p. 38.

6. Voir Paulina Schmidtbauer Rocha: «Institutions psychanalytiques: la politique de l'autruche » (contribution décevante), Joël Birman : « Gouvernabilité, hiérarchies, féminité », et Howard Shevrin :

«La science, la pratique et l'enseignement de la psychanalyse ».

7. «Demeurer révolutionnaires».

8. « Rapports de la psychanalyse au social et au politique ».

9. Auteur d'une très stimulante psychanalyse historique, Le Fantasme Pinochet, Uribe explique notamment pourquoi on peut parler ici d'un « cas » : «Pinochet n'est pas qui. Pinochet est un quoi. » 10. Je passe sur bon nombre de divagations plus ou moins heureuses des uns et des autres (par exemple chez Birman: le colonialisme institutionnel, les pratiques « nazi-fascistes » de l'internationale lacanienne).

11. « Le travail de l'impasse entre la psychanalyse et les neurosciences ». 


\section{Livres en notes}

réductionnisme radical de la biologie et des neurosciences), en insistant sur la grande plasticité des théories actuelles de la complexité et des processus émergents. Certains auteurs osent d'ailleurs chercher concrètement des convergences entre les deux disciplines ${ }^{12}$. En outre, l'ouvrage développe une interrogation lancinante sur la clinique psychanalytique, pour y souligner le rôle non négligeable qu'y joue l'écriture ${ }^{13}$, pour rappeler l'impératif de vérification (à inventer, en montrant l'échec d'hypothèses alternatives), ou pour insister sur sa nécessaire dissociation d'avec la théorie ${ }^{14}$, à des fins de formalisation minimale et de constitution d'un corpus transmissible. Enfin, dans le sillage derridien, la psychanalyse réaffirme son désir de dialogue avec la poésie et la philosophie. Pour Henri Rey-Flaud ${ }^{15}$, notamment, la psychanalyse ne se pose plus face à l'art comme une herméneutique des profondeurs inconscientes (voir le commentaire freudien de la Gradiva), ni un espace de vérification externe des découvertes faites dans le champ clinique (selon un rapport d'application). Elle constitue au contraire pour lui un espace, un lieu, où les questions des autres lieux peuvent trouver une résonance ${ }^{16}$.

Malgré quelques articles franchement décevants, ce petit recueil montre assez que la psychanalyse peut savoir rester une pratique féconde, un « lieu » d'inventions d'outils et de concepts de réflexion.

12. Sur cette politique de la main tendue, que certains ont l'immense mérite de mettre effectivement en pratique (Shevrin), voir René Major, « Du droit à la subjectivité ».

13. Pierre Fedida: «La clinique psychanalytique ».

14. C'est la thèse de la psychanalyse comme science double chez Howard Shevrin, dans son excellente contribution, « La science, la pratique, et l'enseignement de la psychanalyse ».

15. «La psychanalyse, dans son rapport au poétique et à la philosophie ».

16. Voir également la méditation de Ginette Michaud sur l'impossible: «Psychanalyse, littérature, déconstruction : l'impossible en partage », et celle de Derrida: «L'impossible au-delà d'une souveraine cruauté ». 